\title{
Infections à Mycobacterium chimaera chez les patients en phase post-opératoire exposés à des échangeurs thermiques: Un aperçu
}

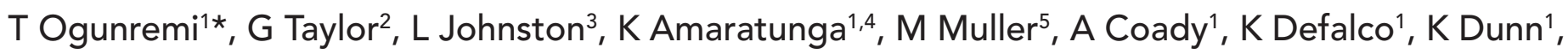 \\ J Johnstone ${ }^{6}$, S Smith², J Embree ${ }^{7}$, B Henry ${ }^{8}$, J Stafford ${ }^{9}$, au nom du groupe de travail d'experts en \\ prévention et en contrôle des infections ${ }^{10}$
}

\section{Résumé}

Une éclosion dans plusieurs pays de l'infection à Mycobacterium chimaera associée à des échangeurs thermiques contaminés a été signalée, avec, à ce jour, plus de 70 cas en Europe et aux États-Unis et deux cas au Canada. Les caractéristiques épidémiologiques et microbiologiques de cette éclosion apportent des données probantes concernant la transmission de source commune de la bactérie $M$. chimaera par l'air d'évacuation des échangeurs thermiques intrinsèquement contaminés aux patients subissant une intervention de chirurgie cardiaque. À ce jour, l'ensemble des cas signalés ont été associés aux échangeurs thermiques Stöckert 3T fabriqués dans une usine de l'entreprise LivaNova avant septembre 2014. L'implantation d'un dispositif de prothèse augmente le risque d'infection. Les infections se présentent généralement sous forme d'endocardite sur prothèse valvulaire, d'infection de greffe vasculaire ou d'infection disséminée. Si les taux de mortalités signalés étaient variables, ils étaient cependant souvent supérieurs à $40 \%$.

Plusieurs mesures sont recommandées pour faciliter la recherche des cas et atténuer le risque d'exposition. La faisabilité de certaines mesures d'atténuation du risque et leur efficacité dans la diminution du risque d'exposition sont encore à déterminer. Tant que les échangeurs thermiques ne seront pas repensés de façon à éviter la contamination de l'eau et l'aérosolisation, la séparation de l'air d'évacuation de ces dispositifs de l'air de la salle d'opération pendant les interventions chirurgicales constituera probablement la stratégie d'atténuation du risque la plus efficace. Toutefois, il convient de prendre en compte les éventuelles conséquences involontaires de cette approche. Cet aperçu résume les conclusions $d$ 'articles d'évaluation par les pairs et d'autres documents nationaux pertinents sur les principales caractéristiques de l'éclosion, notamment la source, les facteurs de risque identifiés de l'infection, les signes et les symptômes de l'infection, le fardeau de la maladie, les mesures d'atténuation des risques, les difficultés de prise en charge et les lacunes en matière de connaissances.

Citation proposée : Ogunremi T, Taylor G, Johnston L, Amaratunga K, Muller M, Coady A, Defalco K, Dunn K, Johnstone J, Smith S, Embree J, Henry B, Stafford J, au nom du groupe de travail d'experts en prévention et en contrôle des infection. Infections à Mycobacterium chimaera chez les patients en phase post-opératoire exposés à des échangeurs thermiques: Un aperçu. Relevé des maladies transmissibles au Canada. 2017;43(5):119-26. https://doi.org/10.14745/ccdr.v43i05a05f

Mots-clés : Mycobacterium chimaera, échangeur thermique, chirurgie cardiaque, circulation extracorporelle

\author{
Affiliations \\ ${ }^{1}$ Centre de la lutte contre les \\ maladies transmissibles et les \\ infections, Agence de la santé \\ publique du Canada, Ottawa \\ (Ontario) \\ ${ }^{2}$ Département de médecine, \\ Université de l'Alberta, Edmonton \\ (Alberta) \\ ${ }^{3}$ Centre des sciences de la \\ santé Queen Elisabeth II et \\ Université Dalhousie, Halifax \\ (Nouvelle-Écosse) \\ ${ }^{4}$ Département de médecine, \\ Université d'Ottawa, Ottawa \\ (Ontario) \\ ${ }^{5}$ Hôpital St. Michael's, Toronto \\ (Ontario) \\ ${ }^{6}$ Santé publique Ontario, Toronto \\ (Ontario) \\ ${ }^{7}$ Université du Manitoba, \\ Winnipeg (Manitoba) \\ ${ }^{8}$ Bureau du médecin-hygiéniste \\ en chef, ministère de la Santé, \\ Victoria (Colombie-Britannique) \\ ${ }^{9}$ Ministère de la Santé, \\ gouvernement du \\ Nouveau-Brunswick, Fredericton \\ (Nouveau-Brunswick) \\ ${ }^{10}$ Tous les collaborateurs du \\ groupe de travail sont mentionnés \\ à la fin du document
}

*Correspondance : toju.

ogunremi@phac-aspc.gc.ca

\section{Introduction}

Les infections associées aux soins de santé liées à la contamination des dispositifs médicaux et à la formation de biofilm ont été documentées dans la littérature (1). Récemment, les échangeurs thermiques utilisés au cours de la circulation extracorporelle, pendant les interventions de chirurgie cardiaque et l'oxygénation extracorporelle ont fait l'objet d'un examen minutieux en raison des infections liées aux dispositifs contaminés $(2,3)$.
Les échangeurs thermiques comportent des réservoirs d'eau qui pompent de l'eau à température contrôlée à travers des circuits fermés dans des échangeurs thermiques externes qui régulent la température corporelle du patient par convection (4). Les dispositifs sont équipés d'un radiateur et d'un ventilateur favorisant la dissipation constante de l'excès de chaleur par les ouvertures de grille et l'agitation de l'eau dans le réservoir donne lieu à une aérosolisation par le biais de l'air d'évacuation $(4,5)$. Les échangeurs thermiques sont soumis à la formation de biofilm. Un 
biofilm est composé d'agrégats de microorganismes intégrés dans une matrice extracellulaire qui adhèrent l'une à l'autre ainsi qu'aux surfaces internes, comme l'intérieur d'un HCD.

Plusieurs types de microorganismes ont été isolés des échangeurs thermiques contaminés, notamment les mycobactéries non tuberculeuses (MNT), omniprésentes dans le sol et l'eau, et liées aux infections associées aux soins de santé (6-9). Les enquêtes menées sur des grappes de cas d'infection par les mycobactéries non tuberculeuses après des interventions de chirurgie cardiaque ont permis de mettre en évidence le microorganisme en cause : Mycobacterium chimaera. Le microorganisme $M$. chimaera est une mycobactérie non tuberculeuse à croissance lente, présente dans le complexe Mycobacterium avium (3,9-12). II est moins susceptible aux procédures de désinfection en raison de ses constituants de la paroi cellulaire et de sa capacité à former des biofilms. L'isolement et l'identification du microorganisme $M$. chimaera dans les échantillons cliniques nécessitent des techniques de microbiologie spécialisées (3). La transmission a été associée à un seul modèle d'échangeur thermique fabriqué par l'entreprise Sorin (maintenant LivaNova) $(3,13)$. Les cultures obtenues à partir des réservoirs d'eau, des circuits d'eau et des échantillons d'air des échangeurs thermiques pendant leur utilisation ont développé le microorganisme $M$. chimaera $(5,8,9,11)$.

Bien qu'une contamination des dispositifs d'oxygénation extracorporelle par M. chimaera ait été signalée, elle ne s'est pas propagée dans l'air de la salle lorsque les dispositifs étaient en cours de fonctionnement et aucune infection à $M$. chimaera associée à l'oxygénation extracorporelle n'a été signalée $(2,14)$. Néanmoins, la nécessité d'évaluer l'exposition éventuelle des patients à ce microorganisme du fait de l'oxygénation extracorporelle a été reconnue. En effet, les patients sous oxygénation extracorporelle, souvent gravement malades et hautement immunodéprimés, peuvent être exposés à ce dispositif pendant une longue période (2). Des documents d'orientation nationale et des communications sur la sécurité décrivant des mesures d'atténuation des risques et des recommandations en matière d'analyses au Canada, au Royaume-Uni, aux États-Unis et en Australie ont été publiés (13,15-20).

L'objectif de cet aperçu est de résumer la documentation pertinente sur l'éclosion actuelle de l'infection à $M$. chimaera dans plusieurs pays. La source d'exposition, les facteurs de risque de I'infection, les signes et les symptômes de l'infection, le fardeau de la maladie, les mesures d'atténuation des risques, les difficultés et les lacunes sont résumés. Cet aperçu peut constituer une ressource utile pour les établissements et les fournisseurs de soins de santé canadiens qui utilisent des échangeurs thermiques. II peut également appuyer la prise de décisions éclairées par les autorités responsables de la mise en œuvre de mesures de prévention et de contrôle des infections.

\section{Portée}

Des recherches documentaires ont été entreprises au niveau mondial par la Bibliothèque de la santé (Santé Canada), dans les bases de données Ovid MEDLINE, EMBASE et Global Health pour les études publiées du 1 1er janvier 2007 au 8 mars 2017. La stratégie de recherche a été élaborée à l'aide d'un lexique propre aux bases de données : "Mycobacterium chimaera ", " échangeurs thermiques " et « chirurgie cardiaque ". La recherche a été limitée aux études en anglais et en français, mais n'a pas été limitée au niveau des types d'étude. Une recherche dans la littérature grise a également été réalisée par la Bibliothèque de la santé dans le but d'identifier des communications sur la sécurité et des documents d'orientation nationale pertinents. Des recherches manuelles ont également été effectuées dans des listes de référence de documents $d$ 'orientation pertinents afin de bénéficier d'études pertinentes supplémentaires.

Les études pertinentes ont été intégralement passées en revue. Objectif : identifier les études faisant état d'infections à $M$. chimaera associées à des échangeurs thermiques chez les patients ayant subi une chirurgie cardiaque (phase post-opératoire) et de mesures d'atténuation des risques. Une synthèse descriptive des publications évaluées par les pairs, des documents d'orientation nationale et/ou des communications sur la sécurité pertinents a été effectuée.

\section{Résultats}

Au total, 95 articles ont été extraits à l'issue des recherches dans les publications évaluées par les pairs et dans la littérature grise, notamment des recherches dans les listes de référence des documents identifiés. Les renseignements de 38 documents pertinents ont été inclus dans cet aperçu. Cinquante-sept articles ont été exclus pour l'une des raisons suivantes : études portant sur des cas déjà décrits en détail dans d'autres sources; études portant sur les mycobactéries non tuberculeuses en général (et non spécifiquement sur M. chimaera); études n'abordant pas l'exposition des patients, ni la transmission; documents d'orientation nationale ou communications sur la sécurité n'apportant pas de renseignements supplémentaires par rapport à ceux obtenus de documents similaires communiqués par le Canada, les États-Unis, l'Australie et l'Europe.

\section{Source d'exposition}

À ce jour, l'ensemble des cas d'infection à $M$. chimaera signalés à l'échelle internationale ont été associés aux échangeurs thermiques Stöckert 3T fabriqués en Allemagne par LivaNova avant septembre 2014 (3,9,13,15,21-23). L'analyse phylogénétique par séquençage du génome entier, entre autres méthodes, a révélé que les isolats de patients infectés, et d'eau et d'air d'évacuation des échangeurs thermiques Stöckert 3T usagés ou neufs étaient étroitement liés, preuve d'une répartition mondiale des échangeurs thermiques contaminés et d'une source commune indépendante du milieu hospitalier de l'éclosion actuelle $(5,9,12,22,24-26)$. L'entreprise LivaNova a apporté des modifications à ses processus de désinfection pour tenter de réduire le risque de contamination par $M$. chimaera des échangeurs thermiques 3T fabriqués après septembre 2014 $(13,15,27,28)$. Des analyses réalisées sur les échangeurs thermiques fabriqués par une autre entreprise ont permis de détecter la présence du microorganisme $M$. chimaera dans l'eau, mais pas dans les échantillons d'air, et l'isolat obtenu était génétiquement différent des isolats obtenus sur les échangeurs thermiques Stöckert 3T $(12,25,29)$.

Pendant les interventions chirurgicales, l'échangeur thermique est souvent positionné à côté de la machine de circulation extracorporelle et du patient. Récemment, dans le cadre des efforts de diminution de l'exposition du patient à l'air d'évacuation de l'échangeur thermique, la faisabilité du positionnement du dispositif immédiatement à côté de la bouche d'évacuation, au niveau du sol, dans la salle d'opération a été étudiée. 


\section{Facteurs de risque de l'infection}

Les cas d'infection à $M$. chimaera après exposition aux échangeurs thermiques pendant la circulation extracorporelle ont été signalés chez des patients ayant subi une intervention chirurgicale en Europe (Royaume-Uni, France, Suisse, Pays-Bas, Allemagne, Irlande et Espagne) ainsi qu'aux États-Unis, en Australie, au Canada et dans la Région administrative spéciale de Hong Kong (18). Les patients subissant une intervention chirurgicale avec circulation extracorporelle, au cours de laquelle leur température corporelle est régulée par un échangeur thermique présentent un risque d'exposition et d'infection (8). Les patients placés sous circulation extracorporelle pendant plus de deux heures avaient plus de risques d'être infectés par les mycobactéries non tuberculeuses (rapport de cotes : 16,5; IC à $95 \%: 3,2-84$ ) (8). Dans les hôpitaux où au moins une infection à $M$. chimaera associée à des échangeurs thermiques a été identifiée, le risque qu'un patient contracte une infection était d'environ 0,1 à $1 \%$ $(11,30,31)$. Sur 115664 interventions chirurgicales réalisées en Angleterre et impliquant la réparation ou le remplacement de valvules cardiaques (entre 2007 et 2014), le risque d'infection par les mycobactéries non tuberculeuses a augmenté de moins de 0,2 cas/10 000 années-personnes avant 2010 à 1,65 cas/10 000 années-personnes en 2013 (29).

L'implantation d'un dispositif de prothèse (par exemple, valvule cardiaque, greffe vasculaire, dispositif d'assistance ventriculaire gauche) augmentait le risque d'infection $(3,11,13,29)$. D'après des données limitées, les greffes cardiaques augmenteraient également le risque d'infection $(3,32)$.

Aucun cas n'est survenu parmi le personnel de salle d'opération exposé à l'aérosolisation des échangeurs thermiques.

\section{Signes et symptômes de l'infection}

Les signes et/ou les symptômes de l'infection invasive à $M$. chimaera contractée après une exposition aux aérosols d'un échangeur thermique peuvent ne pas survenir avant des mois ou des années (après l'exposition). Le délai moyen entre l'exposition et le diagnostic est de 1,6 an (intervalle : 0,1-6,3 ans) $(3,10,14,23,32)$. L'infection se présente généralement sous forme $d^{\prime}$ endocardite sur prothèse valvulaire, d'infection de greffe vasculaire ou d'infection disséminée, bien que plusieurs sites extracardiaques puissent également être infectés (tableau 1) $(9-11,13,18,29,33)$. Les manifestations cliniques de l'infection sont diverses et les symptômes peuvent être non spécifiques $(12,23)$. Dans certains cas, les manifestations extracardiaques ont précédé la maladie cardiovasculaire (11). Une description d'un syndrome compatible avec l'infection par les mycobactéries non tuberculeuses publiée par le Réseau des laboratoires de santé publique du Canada (RLSPC) figure dans le tableau 1 (16).

\section{Fardeau de la maladie}

L'infection à $M$. chimaera requiert un traitement médical agressif en association avec un traitement antimycobactérien et parfois, plusieurs interventions chirurgicales. L'infection entraîne généralement un taux de morbidité important et s'accompagne d'une hospitalisation de longue durée, d'effets indésirables des traitements médicaux et chirurgicaux, et/ou d'un échec du traitement $(3,11,18,29)$. En janvier 2017, en Europe, au moins 52 cas avaient été signalés $(12,18)$. Trois cas ont été identifiés en Australie, vingt-quatre aux États-Unis et deux au Canada $(20,23,32,34)$. Les résultats individuels des patients ne sont pas toujours pris en compte. Selon les données disponibles, la plupart des cas sont survenus chez des adultes plus âgés, bien que l'âge des patients variait de un à 81 ans, dont deux enfants. Environ $83 \%$ des patients étaient des hommes. La plupart des
Tableau 1 : Symptômes cliniques des patients atteints d'une infection à Mycobacterium chimaera

\begin{tabular}{|l|l|}
\hline \multicolumn{1}{|c|}{$\begin{array}{c}\text { Type de } \\
\text { symptômes }\end{array}$} & \multicolumn{1}{c|}{ Symptômes cliniques } \\
\hline Généraux & $\begin{array}{l}\text { Fièvre récurrente ou prolongée, fatigue, } \\
\text { essoufflement, perte de poids, sueurs nocturnes }\end{array}$ \\
\hline Cardiaques & $\begin{array}{l}\text { Endocardite sur prothèse valvulaire et/ou infection } \\
\text { de greffe vasculaire }\end{array}$ \\
\hline Extracardiaques & $\begin{array}{l}\text { Infection osseuse, infection de plaie chirurgicale } \\
\text { de sternotomie, médiastinite, hépatite, infection } \\
\text { du sang, infection oculaire (panuvéite, choroïdite } \\
\text { multifocale, choriorétinite) }\end{array}$ \\
\hline $\begin{array}{l}\text { Immunologiques/ } \\
\text { emboliques }\end{array}$ & Splenomégalie, cytopénie \\
\hline Nourrissons & Épisodes fébriles et retard staturo-pondéral \\
\hline
\end{tabular}

Reproduit avec l'autorisation du Réseau des laboratoires de santé publique du Canada (RLSPC) (16)

études ont fait état d'un taux de mortalité supérieur à $40 \%$ (voir le tableau 2) $(3,11,12,29,32)$ et la mortalité était élevée en cas de retards importants de diagnostic et les patients étaient gravement malades lorsqu'un traitement antimycobactérien approprié a pu être mis en place. La possibilité qu'une sensibilisation accrue et qu'un diagnostic précoce réduisent la mortalité associée à l'infection à $M$. chimaera reste incertaine.

\section{Tableau 2 : Taux de mortalité signalés en lien avec I'infection à Mycobacterium chimaera associée à des échangeurs thermiques}

\begin{tabular}{|c|c|c|}
\hline $\begin{array}{l}\text { Référence } \\
\text { (pays/région) }\end{array}$ & $\begin{array}{l}\text { Nombre de } \\
\text { patients chez qui } \\
\text { l'infection a été } \\
\text { diagnostiquée }\end{array}$ & $\begin{array}{c}\text { Nombre de } \\
\text { décès (taux de } \\
\text { mortalité en \%) }\end{array}$ \\
\hline $\begin{array}{l}\text { Kohler et al., } 2015 \text { (Europe) } \\
\text { (11) }\end{array}$ & $10^{1}$ & $4(40 \%)^{2}$ \\
\hline $\begin{array}{l}\text { Chand et al, } 2016 \text { (Europe) } \\
\text { (29) }\end{array}$ & $18^{3}$ & 9 (50 \%) \\
\hline $\begin{array}{l}\text { Appenheimer et al., } 2016 \\
\text { (États-Unis) (32) }\end{array}$ & 24 & NS $(46 \%)^{4}$ \\
\hline $\begin{array}{l}\text { Centre européen de } \\
\text { prévention et de contrôle } \\
\text { des maladies, } 2016 \\
\text { (Europe) (18) }\end{array}$ & $52^{5}$ & $10(<19 \%)^{6}$ \\
\hline $\begin{array}{l}\text { Haller et al., } 2016 \\
\text { (Allemagne) (9) }\end{array}$ & 5 & $1(20 \%)^{7}$ \\
\hline $\begin{array}{l}\text { Tan et al., } 2016 \text { (États-Unis) } \\
\text { (33) }\end{array}$ & 3 & $2(67 \%)^{6,7}$ \\
\hline $\begin{array}{l}\text { Public Health England, } \\
2017 \text { (Europe) (12) }\end{array}$ & 26 & 15 (58 \%) \\
\hline $\begin{array}{l}\text { Australian Commission } \\
\text { on Safety and Quality } \\
\text { in Health Care, } 2017 \\
\text { (Australie) (20) }\end{array}$ & 3 & 0 (0 \%) \\
\hline \multicolumn{3}{|c|}{$\begin{array}{l}\text { Abréviations : NS, non signalé; \%, pourcentage } \\
{ }^{1} \text { Neuf cas ont été confirmés; un cas était probable } \\
{ }^{2} \text { Un décès supplémentaire n'était pas lié à l'infection à M. chimaera } \\
{ }^{3} \text { Tous les cas étaient probables } \\
{ }^{4} \text { Le nombre de décès n'a pas été signalé } \\
{ }^{5} \text { Certains de ces cas ont été signalés dans d'autres publications } \\
{ }^{\circ} \text { Cause des décès non décrite ou les décès n'ont pas tous été attribués à l'infection } \\
7 \text { Le taux de mortalité n'a pas été signalé dans l'étude; il a été calculé pour être présenté dans ce } \\
\text { tableau }\end{array}$} \\
\hline
\end{tabular}

RMTC・ Le 4 mai $2017 \bullet$ Volume 43-5

Page 121 


\section{Mesures d'atténuation des risques}

Les principales mesures identifiées pour faciliter la recherche des cas et atténuer l'exposition à venir au microorganisme M. chimaera sont résumées dans le tableau 3.

Tableau 3 : Mesures recommandées pour faciliter la recherche des cas et atténuer l'exposition à venir au microorganisme Mycobacterium chimaera

\begin{tabular}{|c|c|}
\hline $\begin{array}{l}\text { Mesure } \\
\text { d'atténuation } \\
\text { des risques }\end{array}$ & Contexte supplémentaire et/ou limites \\
\hline $\begin{array}{l}\text { Formation et } \\
\text { notification des } \\
\text { fournisseurs de soins } \\
\text { de santé }(11,12,28,32)\end{array}$ & $\begin{array}{l}\text { - Les cas ont été détectés grâce à la notification } \\
\text { des fournisseurs. } \\
\text { - La première intervention chirurgicale en cause a } \\
\text { - Maintentectuée en } 2007 \text {. } \\
\text { concernant l'infection à M. chimaera ou } \\
\text { d'autres infections par les mycobactéries non } \\
\text { tuberculeuses chez les patients (qui ont subi } \\
\text { une intervention chirurgicale avec circulation } \\
\text { extracorporelle et échangeurs thermiques } \\
\text { entre } 2007 \text { et la mise en œuvre des mesures } \\
\text { d'atténuation des risques). }\end{array}$ \\
\hline $\begin{array}{l}\text { Notification des } \\
\text { patients }(8,12,28,32)\end{array}$ & $\begin{array}{l}\text { - À ce jour, aucun cas n'a été identifié par le biais } \\
\text { de la notification des patients. } \\
\text { - Les analyses ne sont pas recommandées dans le } \\
\text { cas d'individus exposés asymptomatiques. } \\
\text { - Tant que les mesures d'atténuation des risques } \\
\text { ne sont pas mises en œuvre, les renseignements } \\
\text { relatifs à une exposition potentielle devraient être } \\
\text { communiqués aux patients avant l'intervention } \\
\text { chirurgicale. }\end{array}$ \\
\hline $\begin{array}{l}\text { Amélioration de } \\
\text { la surveillance } \\
\text { prospective des } \\
\text { mycobactéries non } \\
\text { tuberculeuses }(9,21)\end{array}$ & $\begin{array}{l}\text { - Le Centre européen de prévention et de contrôle } \\
\text { des maladies (ECDC) a publié un protocole de } \\
\text { détection des cas. }\end{array}$ \\
\hline $\begin{array}{l}\text { Assurer la traçabilité } \\
\text { des échangeurs } \\
\text { thermiques utilisés } \\
\text { (12) }\end{array}$ & $\begin{array}{l}\text { - Les unités individuelles utilisées au cours de } \\
\text { chaque intervention chirurgicale devraient être } \\
\text { enregistrées au cas où une infection se produirait } \\
\text { ultérieurement. }\end{array}$ \\
\hline $\begin{array}{l}\text { Retirer les échangeurs } \\
\text { thermiques } \\
\text { potentiellement } \\
\text { contaminés de la } \\
\text { circulation }(12,15,27)\end{array}$ & $\begin{array}{l}\text { - Dans la mesure du possible, l'ensemble des } \\
\text { échangeurs thermiques Stöckert 3T fabriqués par } \\
\text { LivaNova avant septembre } 2014 \text { devraient être } \\
\text { retirés de la circulation. } \\
\text { - Dans certains contextes, les risques liés au report } \\
\text { de l'intervention chirurgicale sont supérieurs aux } \\
\text { risques associés à la réalisation de l'intervention } \\
\text { avec des échangeurs thermiques dont la } \\
\text { contamination est confirmée ou soupçonnée. }\end{array}$ \\
\hline $\begin{array}{l}\text { Remplacer les } \\
\text { échangeurs } \\
\text { thermiques } \\
\text { contaminés, ainsi } \\
\text { que les accessoires, } \\
\text { les tubes et les } \\
\text { raccords, pour éviter } \\
\text { une recontamination } \\
(13,15,27,35)\end{array}$ & $\begin{array}{l}\text { - L'entreprise LivaNova a mis en œuvre un } \\
\text { programme pour proposer aux utilisateurs, } \\
\text { dans certaines circonstances, un prêt de } \\
\text { dispositif afin de leur permettre de poursuivre } \\
\text { l'intervention pendant la désinfection profonde } \\
\text { de leur dispositif. La demande internationale } \\
\text { d'échangeurs thermiques de remplacement } \\
\text { peut donner lieu à des retards dans } \\
\text { l'approvisionnement. }\end{array}$ \\
\hline $\begin{array}{l}\text { Utiliser le protocole } \\
\text { de fonctionnement du } \\
\text { fabricant, notamment } \\
\text { les procédures de } \\
\text { nettoyage et de } \\
\text { désinfection à jour. } \\
(3,9,12,15,27,28,35)\end{array}$ & $\begin{array}{l}\text { - Tenir un journal de nettoyage et de désinfection } \\
\text { des échangeurs thermiques. } \\
\text { - Consulter régulièrement le site Web du fabricant } \\
\text { pour connaître les mises à jour applicables. } \\
\text { - Les protocoles de décontamination actuels } \\
\text { doivent encore être validés. Les études ont } \\
\text { contesté l'efficacité de ces protocoles, mettant } \\
\text { en évidence l'échec systématique de la } \\
\text { décontamination. L'élimination des biofilms est } \\
\text { essentielle pour une décontamination efficace } \\
\text { des échangeurs thermiques. }\end{array}$ \\
\hline
\end{tabular}

Tableau 3 : Mesures recommandées pour faciliter la recherche des cas et atténuer l'exposition à venir au microorganisme Mycobacterium chimaera (suite)

\begin{tabular}{|c|c|}
\hline $\begin{array}{l}\text { Mesure d'atténuation } \\
\text { des risques (suite) }\end{array}$ & $\begin{array}{c}\text { Contexte supplémentaire et/ou } \\
\text { limites (suite) }\end{array}$ \\
\hline $\begin{array}{l}\text { Réalisation d'analyses } \\
\text { microbiologiques } \\
\text { régulières sur les } \\
\text { échangeurs thermiques } \\
\text { utilisés }(12,15,17,25,27,36)\end{array}$ & $\begin{array}{l}\text { - Cette mesure n'a pas été adoptée } \\
\text { à grande échelle en raison du taux } \\
\text { élevé de faux-négatifs et du manque } \\
\text { de méthodes validées et normalisées } \\
\text { pour le prélèvement et le traitement } \\
\text { des échantillons, et la détection du } \\
\text { microorganisme M. chimaera. } \\
\text { - Le Réseau des laboratoires de santé } \\
\text { publique du Canada (RLSPC) et la } \\
\text { Food and Drug Administration (FDA) } \\
\text { des États-Unis déconseillent de réaliser } \\
\text { de mises en culture environnementales } \\
\text { régulières à partir des échangeurs } \\
\text { thermiques pour détecter la présence } \\
\text { du microorganisme M. chimaera. }\end{array}$ \\
\hline $\begin{array}{l}\text { Recourir à des solutions } \\
\text { techniques pour permettre } \\
\text { une séparation fiable de } \\
\text { l'air d'évacuation des } \\
\text { échangeurs thermiques de } \\
\text { l'air de la salle d'opération } \\
(4,5,12,13,15,18,25,26,37)\end{array}$ & $\begin{array}{l}\text { Voici les solutions possibles : } \\
\text { - Placer l'échangeur thermique en } \\
\text { dehors de la salle d'opération, tubes } \\
\text { connectés à travers une ouverture } \\
\text { dans le mur (en veillant à ce que la } \\
\text { pression positive de l'air à l'intérieur } \\
\text { de la salle d'opération soit maintenue). } \\
\text { Bien qu'il s'agisse de la solution la plus } \\
\text { fiable, les conséquences involontaires } \\
\text { (par exemple, une circulation de } \\
\text { l'air éventuellement modifiée dans } \\
\text { la salle d'opération et une plus } \\
\text { grande distance entre l'échangeur } \\
\text { thermique et le champ opératoire) sont } \\
\text { inconnues. } \\
\text { - Enfermer l'échangeur thermique } \\
\text { dans un boîtier sur mesure avec } \\
\text { ventilation à part (par exemple, reliée } \\
\text { au conduit d'évacuation de la salle } \\
\text { d'opération). Les raccordements } \\
\text { à l'échangeur thermique peuvent } \\
\text { nécessiter l'autorisation du fabricant. } \\
\text { Les conséquences involontaires de } \\
\text { cette solution (par exemple, les effets } \\
\text { du boîtier sur mesure sur le bon } \\
\text { fonctionnement du dispositif) sont } \\
\text { inconnues. } \\
\text { S'il est impossible de séparer de } \\
\text { façon sûre l'air d'évacuation de } \\
\text { l'échangeur thermique de l'air de } \\
\text { la salle d'opération, déplacer le } \\
\text { dispositif le plus loin possible (de } \\
\text { préférence à plus de cinq mètres) } \\
\text { du champ opératoire, l'évacuation } \\
\text { orientée loin du patient et des } \\
\text { instruments exposés, et, si possible, } \\
\text { placer l'échangeur thermique près } \\
\text { de l'évacuation d'air de la salle. Des } \\
\text { expériences de dispersion des fumées } \\
\text { ont démontré que l'air d'évacuation } \\
\text { des échangeurs thermiques était } \\
\text { propulsé pour fusionner avec la } \\
\text { circulation d'air ultrapropre près du } \\
\text { plafond de la salle d'opération. Par } \\
\text { conséquent, il n'est pas certain que } \\
\text { cette approche soit utile dans la } \\
\text { séparation de l'air d'évacuation des } \\
\text { échangeurs thermiques de l'air de la } \\
\text { salle d'opération (4). }\end{array}$ \\
\hline
\end{tabular}

Abréviations : ECDC, Centre européen de prévention et de contrôle des maladies; FDA, Food and Drug Administration des États-Unis 


\section{Difficultés et lacunes}

Le tableau 4 résume les difficultés et les lacunes relatives aux données probantes éclairant la prise en charge clinique de I'infection à $M$. chimaera.

Tableau 4 : Difficultés et lacunes relatives aux données probantes éclairant la prise en charge de l'infection à Mycobacterium chimaera

\begin{tabular}{|c|c|}
\hline Difficulté/lacune & Contexte supplémentaire \\
\hline $\begin{array}{l}\text { L'ampleur du risque } \\
\text { d'infection à } M \text {. chimaera } \\
\text { et l'importance de } \\
\text { l'éclosion sont inconnues } \\
(12,14,29)\end{array}$ & $\begin{array}{l}\text { - Une forte prévalence du microorganisme } \\
\text { M. chimaera sur les échangeurs thermiques } \\
\text { a été signalée (jusqu'à } 80 \% \text { au Danemark). } \\
\text { - Le risque d'infection des patients semble } \\
\text { actuellement faible; toutefois, si une } \\
\text { infection survient, son incidence sur le } \\
\text { patient peut être grave. } \\
\text { - Le risque d'infection et de présentation } \\
\text { clinique parmi la population de patients } \\
\text { pédiatriques est inconnu. }\end{array}$ \\
\hline $\begin{array}{l}\text { Retard dans l'apparition } \\
\text { des symptômes et le } \\
\text { diagnostic de l'infection } \\
(3,10,14,23,32)\end{array}$ & $\begin{array}{l}\text { - Le délai décrit entre l'exposition et le } \\
\text { diagnostic était compris entre } 0,1 \text { et } 6,3 \text { ans } \\
\text { (moyenne de 1,6 an). } \\
\text { - Peu de laboratoires sont équipés } \\
\text { pour mettre en culture et identifier le } \\
\text { microorganisme } M \text {. chimaera, ce qui peut } \\
\text { contribuer à un retard dans le diagnostic. } \\
\text { - Le développement lent de la culture } \\
\text { de M. chimaera contribue au retard de } \\
\text { diagnostic. } \\
\text { - Le prélèvement précoce d'un milieu de } \\
\text { culture mycobactérien dédié peut accélérer } \\
\text { le diagnostic par rapport aux délais courants. }\end{array}$ \\
\hline $\begin{array}{l}\text { Efficacité et effets } \\
\text { indésirables du traitement } \\
(3,11,29,32)\end{array}$ & $\begin{array}{l}\text { - L'infection à } M \text {. chimaera peut être très } \\
\text { difficile à traiter en raison de la résistance } \\
\text { intrinsèque du microorganisme à de } \\
\text { nombreux agents antimicrobiens, de sa } \\
\text { tendance à former des biofilms sur les } \\
\text { dispositifs implantés, du site profond de } \\
\text { l'infection, compliquant la pénétration des } \\
\text { antimicrobiens (par exemple, endocardite, } \\
\text { infection de greffe et infection osseuse). } \\
\text { - Le traitement est prolongé et nécessite une } \\
\text { combinaison d'agents antimicrobiens. } \\
\text { - L'infection disséminée a souvent nécessité } \\
\text { plusieurs interventions chirurgicales, avec } \\
\text { des taux de mortalité élevés. }\end{array}$ \\
\hline $\begin{array}{l}\text { La mise au point de } \\
\text { nouveaux modèles } \\
\text { d'échangeurs thermiques } \\
\text { est en cours }(5,15)\end{array}$ & $\begin{array}{l}\text { - L'élaboration de conteneurs sur mesure } \\
\text { dotés de filtres HEPA pour accueillir les } \\
\text { échangeurs thermiques ne pouvant pas être } \\
\text { placés à l'extérieur de la salle d'opération } \\
\text { est en cours, mais leur efficacité est } \\
\text { actuellement inconnue. } \\
\text { - Les fabricants d'échangeurs thermiques } \\
\text { sont en train de modifier leurs modèles } \\
\text { pour limiter l'aérosolisation et prévenir la } \\
\text { transmission. }\end{array}$ \\
\hline $\begin{array}{l}\text { L'ampleur des infections } \\
\text { associées aux échangeurs } \\
\text { thermiques causées par } \\
d^{\prime} \text { 'autres microorganismes } \\
\text { tels que l'espèce } \\
\text { Legionella est inconnue } \\
(12,29)\end{array}$ & $\begin{array}{l}\text { - La surveillance à l'échelle nationale } \\
\text { au Royaume-Uni (2007-2016) n'a pas } \\
\text { permis d'identifier de cas de maladie du } \\
\text { légionnaire chez les travailleurs de la santé } \\
\text { éventuellement exposés dans le cadre de } \\
\text { leur activité professionnelle aux échangeurs } \\
\text { thermiques. } \\
\text { - Aucune endocardite due à l'espèce } \\
\text { Legionella n'a été signalée chez les patients } \\
\text { ayant subi une chirurgie cardiaque (phase } \\
\text { post-opératoire) pendant cette éclosion. }\end{array}$ \\
\hline
\end{tabular}

Abréviation : HEPA, filtre à haute efficacité pour les particules de l'air

\section{Discussion}

Les résultats de cet aperçu mettent en évidence un risque faible mais accru d'infection à $M$. chimaera avec l'utilisation des échangeurs thermiques contaminés par une source commune pendant la circulation extracorporelle (29). Au vu de la longue période de latence, on s'attend à ce que des cas supplémentaires se produisent. L'ampleur réelle des risques après exposition est incertaine; les estimations actuelles sont basées sur des données très limitées. Néanmoins, les risques liés au report de l'intervention de chirurgie cardiaque sont généralement considérés nettement supérieurs aux risques associés à cette infection, même lorsque le risque d'infection n'a pas été entièrement atténué (28).

Il est possible d'éviter l'exposition à venir des patients en mettant en œuvre des mesures d'atténuation des risques, notamment en utilisant des échangeurs thermiques non contaminés ou en remplaçant les dispositifs contaminés le plus tôt possible. La recherche des cas peut être accélérée par la mise au point de tests de polymérase en chaîne pour la détection rapide et fiable du microorganisme $M$. chimaera dans les échantillons cliniques ou environnementaux.

Il est urgent d'améliorer les modèles des échangeurs thermiques afin de favoriser une décontamination fiable et d'éviter que les aérosols atteignent le champ opératoire $(5,11)$. Ces améliorations peuvent nécessiter une consultation et une collaboration entre les fabricants des dispositifs médicaux, les ingénieurs et les experts en prévention et contrôle des infections.

Cet aperçu est limité par l'insuffisance des données pour estimer l'ampleur réelle du risque d'infection, ainsi que l'absence de données sur l'efficacité et la faisabilité des mesures d'atténuation des risques.

\section{Conclusion}

Les caractéristiques épidémiologiques et microbiologiques de cette éclosion apportent des données probantes concernant la transmission aux patients de la bactérie $M$. chimaera par l'air d'évacuation des échangeurs thermiques Stöckert 3T contaminés, pendant la circulation extracorporelle, contamination ayant donné lieu à une endocardite, une infection du site opératoire et/ou une infection disséminée. L'ampleur réelle des risques après exposition est incertaine; les estimations actuelles sont basées sur des données très limitées.

La séparation de l'air d'évacuation des échangeurs thermiques de l'air de la salle d'opération pendant les interventions chirurgicales constitue probablement la stratégie d'atténuation des risques la plus efficace. La faisabilité de la mise en œuvre des mesures d'atténuation des risques actuellement recommandées est encore à déterminer. En outre, il est nécessaire de réaliser des études pour déterminer les éventuelles conséquences involontaires de la mise en œuvre de ces mesures. L'élaboration de nouveaux modèles des échangeurs thermiques hermétiques et/ou non susceptibles de favoriser la formation de biofilms peut résoudre ce problème. 


\section{Déclaration des auteurs}

T.O. - Administration du projet, conceptualisation, méthodologie, recherche, rédaction et résumé des données (préparation de la version originale, examen et révision); G.T., L.J., M.M. - Expertise clinique, contenu scientifique, rédaction, examen et révision; K.A. - Expertise clinique, contenu scientifique, examen et révision; A.C., K. Defalco - Examen des données résumées, recherche, examen et révision; K. Dunn Conceptualisation, supervision, examen et révision; J.J., S.S., J.E., B.H., J.S. - Expertise clinique, contenu scientifique, examen et révision.

\section{Conflit d'intérêts}

Aucun.

\section{Collaborateurs}

Les auteurs souhaitent remercier les membres suivants du groupe de travail d'experts en prévention et en contrôle des infections pour leur contribution aux discussions sur le contenu de cet article :

Gwen Cerkowniak, Région sanitaire de Saskatoon, Saskatoon (Saskatchewan)

Maureen Cividino, Santé publique Ontario, Toronto (Ontario) Della Gregoraschuk, Alberta Health Services, Edmonton (Alberta)

Patsy Rawding, Autorité sanitaire de la Nouvelle-Écosse, Wolfville (Nouvelle-Écosse)

Sandra Savery, Centre de santé et services sociaux des Sommets, Sainte-Agathe-des-Monts (Québec)

Heidi Pitfield, Unité de santé du district de Simcoe Muskoka, Barrie (Ontario)

Patrice Savard, Centre hospitalier de l'Université de Montréal, Montréal (Québec)

\section{Remerciements}

Les auteurs souhaitent remercier le Réseau des laboratoires de santé publique du Canada (RLSPC) pour les renseignements fournis, présentés dans le tableau 1 de cet article.

Les auteurs remercient également Lynda Gamble (Bibliothèque de la santé, Santé Canada), qui a dirigé les travaux de recherche documentaire pour cet aperçu; Caroline M. Desjardins (Agence de la santé publique du Canada) pour avoir mentionné et produit des références pour cet article; et la $D^{\text {re }}$ Margaret Gale-Rowe (Agence de la santé publique du Canada) pour avoir examiné la version finale de l'article.

\section{Financement}

Ce travail a été appuyé par l'Agence de la santé publique du Canada.

\section{Références}

1. Percival SL, Suleman L, Vuotto C, Donelli G. Healthcareassociated infections, medical devices and biofilms: risk, tolerance and control. J Med Microbiol [Internet]. 2015 [consulté le 16 mars 2017];64(Pt 4):323-34. Disponible à l'adresse : http://jmm.microbiologyresearch.org/content/ journal/jmm/10.1099/jmm.0.000032\#tab2.

2. Trudzinski FC, Schlotthauer U, Kamp A, Hennemann K, Muellenbach RM, Reischl U, Gärtner B, Wilkens H, Bals R, Herrmann M, Lepper PM, Becker SL. Clinical implications of Mycobacterium chimaera detection in thermoregulatory devices used for extracorporeal membrane oxygenation (ECMO), Germany, 2015 to 2016. Euro Surveill [Internet]. 2016 [consulté le 16 mars 2017];21(46):1-8. Disponible à l'adresse : http://www.eurosurveillance.org/ViewArticle. aspx?Articleld=22641.

3. Sommerstein R, Schreiber PW, Diekema DJ, Edmond MB, Hasse B, Marschall J, Sax H. Mycobacterium chimaera outbreak associated with heater-cooler devices: piecing the puzzle together. Infect Control Hosp Epidemiol [Internet]. 2017 [consulté le 16 mars 2017];38(1):103-8. Disponible à l'adresse : https://www.cambridge.org/core/ journals/infection-control-and-hospital-epidemiology/ article/div-classtitlespan-classitalicmycobacteriumchimaeraspan-outbreak-associated-with-heater-coolerdevices-piecing-the-puzzle-togetherdiv/5E98B996F0OA04AD46FFD74C8513B6D1.

4. Sommerstein R, Ruegg C, Kohler P, Bloemberg G, Kuster SP, Sax $\mathrm{H}$. Transmission of Mycobacterium chimaera from heatercooler units during cardiac surgery despite an ultraclean air ventilation system. Emerg Infect Dis [Internet]. 2016 [consulté le 16 mars 2017];22(6):1008-13. Disponible à l'adresse : http://wwwnc.cdc.gov/eid/article/22/6/16-0045_article.

5. Sax H, Bloemberg G, Hasse B, Sommerstein R, Kohler P, Achermann Y, Rössle M, Falk V, Kuster SP, Böttger EC, Weber R. Prolonged outbreak of Mycobacterium chimaera infection after open-chest heart surgery. Clin Infect Dis [Internet]. 2015 [consulté le 16 mars 2017];61(1):67-75. Disponible à l'adresse : http://cid.oxfordjournals.org/content/61/1/67. long.

6. Behr M, Jarand J, Marras TK. Normes Canadiennes pour la lutte antituberculeuse $7^{\mathrm{e}}$ édition [Internet]. Ottawa (ON): Agence de la santé publique du Canada; 2014 [consulté le 16 mars 2017]. Disponible à l'adresse : http://www.phacaspc.gc.ca/tbpc-latb/pubs/tb-canada-7/tb-standards-tbnormes-ch11-fra.php.

7. Galassi L, Donato R, Tortoli E, Burrini D, Santianni D, Dei R. Nontuberculous mycobacteria in hospital water systems: application of HPLC for identification of environmental mycobacteria. J Water Health [Internet]. 2003 [consulté le 16 mars 2017];1(3):133-9. Disponible à l'adresse : http://jwh. iwaponline.com/content/1/3/133.

8. Lyman M, Grigg C, Kinsey CB, Keckler MS, MoultonMeissner H, Noble-Wang J, Miller JR, Perz JF, Perkins K. Invasive nontuberculous mycobacteria infections among cardiothoracic surgery patients - Hospital A, Pennsylvania, 2010-2015. 65th Annual Epidemic Intelligence Service (EIS) Conference. 2016 [consulté le 16 mars 2017]:94. Disponible 
à l'adresse : http://www.cdc.gov/eis/downloads/eisconference-2016.pdf.

9. Haller S, Holler C, Jacobshagen A, Hamouda O, Abu SM., Monnet DL, Plachouras D, Eckmanns T. Contamination during production of heater-cooler units by Mycobacterium chimaera potential cause for invasive cardiovascular infections: results of an outbreak investigation in Germany, April 2015 to February 2016. Euro Surveill [Internet]. 2016 [consulté le 16 mars 2017];21(17). Disponible à I'adresse : http://www.eurosurveillance.org/ViewArticle. aspx?Articleld=22461.

10. Achermann $Y$, Rossle $M$, Hoffmann M, Deggim $V$, Kuster S, Zimmermann DR, Bloemberg G, Hombach M, Hasse B. Prosthetic valve endocarditis and bloodstream infection due to Mycobacterium chimaera. J Clin Microbiol [Internet]. 2013 [consulté le 16 mars 2017];51(6):1769-73. Disponible à I'adresse : http://jcm.asm.org/content/51/6/1769.long.

11. Kohler P, Kuster SP, Bloemberg G, Schulthess B, Frank M, Tanner FC, Rössle M, Böni C, Falk V, Wilhelm MJ, Sommerstein R, Achermann Y, Ten Oever J, Debast SB, Wolfhagen MJ, Brandon Bravo Bruinsma GJ, Vos MC, Bogers A, Serr A, Beyersdorf F, Sax H, Böttger EC, Weber $R$, van Ingen J, Wagner D, Hasse B. Healthcare-associated prosthetic heart valve, aortic vascular graft, and disseminated Mycobacterium chimaera infections subsequent to open heart surgery. Eur Heart J [Internet]. 2015 [consulté le 16 mars 2017];36(40):2745-53. Disponible à l'adresse : http:// eurheartj.oxfordjournals.org/content/36/40/2745.long.

12. Public Health England. Infections associated with heater cooler units used in cardiopulmonary bypass and ecmo: information for healthcare providers in the UK, version 2 [Internet]. London: Public Health England; 2017 [consulté le 16 mars 2017]. Disponible à l'adresse : http://www.gov.uk/ government/publications/infections-associated-with-heatercooler-units-used-in-cardiopulmonary-bypass-and-ecmo.

13. UPDATE: Mycobacterium chimaera infections associated with LivaNova PLC (formerly Sorin Group Deutschland $\mathrm{GmbH}$ ) Stöckert 3T Heater-Cooler System: FDA Safety Communication [Internet]. Silver Spring (MD): U.S.: Food and Drug Administration [mise à jour le 13 oct 2016; consulté le 16 mars 2017]. Disponible à l'adresse : http://www.fda.gov/ MedicalDevices/Safety/AlertsandNotices/ucm520191.htm.

14. Struelens MJ, Plachouras D. Mycobacterium chimaera infections associated with heater-cooler units (HCU): closing another loophole in patient safety. Euro Surveill [Internet]. 2016 [consulté le 16 mars 2017];21(46):1-3. Disponible à I'adresse : http://www.eurosurveillance.org/ViewArticle. aspx?Articleld $=22645$.

15. Échangeur thermique Stöckert 3T de LivaNova - Risque d'infection à Mycobacterium chimaera [Internet]. Ottawa (ON): Santé Canada; 2017 [consulté le 16 mars 2017]. Disponible à l'adresse : http://canadiensensante.gc.ca/recallalert-rappel-avis/hc-sc/2017/62252a-fra.php.

16. Antonation K, Patel S, Trumble Waddell J, Poliquin G, Alexander DC, Hoang L, Farrell D, Garceau R, Haldane D, Jamieson F, Marchand, R, MacKeen A, Marcino D, Theriault S, Tyrrell GJ, Zahariadis G, Zelyas N. Lignes directrices provisoires pour les analyses effectuées en laboratoire visant la détection des infections à Mycobacterium non tuberculeuses (MNT) chez les patients en phase postopératoire exposés à des échangeurs thermiques. Relevé des maladies transmissibles au Canada [Internet]. 2017 [consulté le 16 mars 2017];43(1):27-31. Disponible à l'adresse : http:// www.phac-aspc.gc.ca/publicat/ccdr-rmtc/17vol43/dr-rm43-1/ ar-05-fra.php.

17. Réseau des laboratoires de Santé publique du Canada (CPHLN) Groupe de travail sur la vérification des instruments. Recommandations du Groupe de travail sur la vérification des instruments du Réseau des laboratoires de santé publique du Canada au sujet de la contamination d'échangeurs thermiques par des mycobactéries non tuberculeuses [Internet]. Ottawa (ON): Gouvernement du Canada [mise à jour le 9 fév 2017; consulté le 16 mars 2017]. Disponible à l'adresse : https://www.canada.ca/fr/ sante-publique/services/publications/maladies-et-affections/ verification-instruments-contamination-echangeursthermiques-mycobacteries-non-tuberculeuses.html.

18. European Centre for Disease Prevention and Control (ECDC). Rapid risk assessment: Invasive cardiovascular infection by Mycobacterium chimaera associated with the 3T heatercooler system used during open-heart surgery [Internet]. Stockholm (SE): ECDC; 2016 [consulté le 2017 Mar 16]. Disponible à l'adresse : http://www.ecdc.europa.eu/en/ publications/Publications/RRA-mycobacterium-chimaeraNovember-2016.pdf.

19. Centers for Disease Control and Prevention. Non-tuberculous Mycobacterium (NTM) infections and heater-cooler devices interim practical guidance: Updated October 27, 2015 [Internet]. Atlanta (GA): CDC [mise à jour le 27 oct 2015; consulté le 2017 Mar 16]. Disponible à l'adresse : www.cdc. gov/HAl/pdfs/outbreaks/CDC-Notice-Heater-Cooler-Unitsfinal-clean.pdf.

20. National infection control guidance for non-tuberculous Mycobacterium associated with heater-cooler devices [Internet]. Sydney (AUS): Australian Commission on Safety and Quality in Health Care; 2017 [consulté le 16 mars 2017]. Disponible à l'adresse: https://www.safetyandquality.gov. au/publications/national-infection-control-guidance-for-nontuberculous-mycobacterium-associated-with-heater-coolerdevices/.

21. European Centre for Disease Prevention and Control (ECDC). EU protocol for case detection, laboratory diagnosis and environmental testing of Mycobacterium chimaera infections potentially associated with heater-cooler units: case definition and environmental testing methodology [Internet]. Stockholm (SE): ECDC; 2015 [consulté le 16 mars 2017]. Disponible à l'adresse : http://ecdc.europa.eu/en/ publications/Publications/EU-protocol-for-M-chimaera.pdf.

22. Williamson D, Howden B, Stinear T. Mycobacterium chimaera spread from heating and cooling units in heart surgery. N Engl J Med. 2017;376(6):600-2. Disponible à l'adresse : http://www.nejm.org/doi/full/10.1056/ NEJMc1612023.

23. Bursle E, Playford EG, Coulter C, Griffin P. First Australian case of disseminated Mycobacterium chimaera infection post-cardiothoracic surgery. Infect Dis Health; Forthcoming 2017. Disponible à l'adresse : http://www.sciencedirect.com/ science/article/pii/S2468045117300020. 
24. Perkins KM, Lawsin A, Hasan NA, Strong M, Halpin AL, Rodger RR, Moulton-Meissner H, Crist MB, Schwartz S, Marders J, Daley CL, Salfinger M, Perz JF. Notes from the Field: Mycobacterium chimaera contamination of heatercooler devices used in cardiac surgery - United States. MMWR Morb Mortal Wkly Rep [Internet]. 2016 [consulté le 2017 Mar 16];65(40):1117-8. Disponible à l'adresse : http:// www.cdc.gov/mmwr/volumes/65/wr/mm6540a6.htm.

25. Svensson E, Jensen ET, Rasmussen EM, Folkvardsen DB, Norman A, Lillebaek T. Mycobacterium chimaera in heater-cooler units in Denmark related to isolates from the United States and United Kingdom. Emerg Infect Dis. 2017;23(3):507-9. Disponible à l'adresse : https://wwwnc.cdc. gov/eid/article/23/3/16-1941_article.

26. Schreiber PW, Kuster SP, Hasse B, Bayard C, Ruegg C, Kohler P, Keller PM, Bloemberg GV, Maisano F, Bettex D, Halbe M, Sommerstein R, Sax H. Reemergence of Mycobacterium chimaera in heater-cooler units despite intensified cleaning and disinfection protocol. Emerg Infect Dis. 2016;22(10):1830-3. Disponible à l'adresse : http://wwwnc. cdc.gov/eid/article/22/10/16-0925_article.

27. Sorin Group. Sorin 3T Heater-Cooler System [Internet] [mise à jour en 2016; consulté le 16 mars 2017]. Disponible à I'adresse : http://www.livanova.sorin.com/products/cardiacsurgery/perfusion/h/m/3t.

28. Kanamori H, Weber DJ, Rutala WA. Healthcare-associated Mycobacterium chimaera transmission and infection prevention challenges: Role of heater-cooler units as a water source in cardiac surgery. Clin Infect Dis. 2017;64(3):3436. Disponible à l'adresse : http://cid.oxfordjournals.org/ content/early/2016/12/23/cid.ciw755.full.pdf+html.

29. Chand M, Lamagni T, Kranzer K, Hedge J, Moore G, Parks S, Collins S, del Ojo Elias C, Ahmed N, Brown T, Smith EG, Hoffman P, Kirwan P, Mason B, Smith-Palmer A, Veal P, Lalor MK, Bennett A, Walker J, Yeap A, Isidro Carrion Martin A, Dolan G, Bhatt S, Skingsley A, Charlett A, Pearce D, Russell K, Kendall S, Klein AA, Robins S, Schelenz S, Newsholme W, Thomas S, Collyns T, Davies E, McMenamin J, Doherty L, Peto TE, Crooke D, Zambon M, Phin N. Insidious risk of severe Mycobacterium chimaera infection in cardiac surgery patients. Clin Infect Dis. 2016;64(3):335-42. Disponible à l'adresse : http://cid.oxfordjournals.org/content/ early/2016/12/05/cid.ciw754.full.pdf+html.

30. Centers for Disease Control and Prevention. Contaminated devices putting open-heart surgery patients at risk [Internet]. Atlanta (GA): CDC [mise à jour le 13 oct 2016; consulté le 16 mars 2017]. Disponible à l'adresse : http://www.cdc.gov/ media/releases/2016/p1013-contaminated-devices-.html.
31. Miller MM, Baer L, Freer $C$, Hnatuck $P$, Julian KG, Mincemoyer S, Snyder R, Whitener C. Mycobacterium chimaera infections following open chest cardiac surgery Pennsylvania. Am J Infect Control. 2016;44 Suppl(6 Suppl):S26-7. Disponible à l'adresse : http://www. ajicjournal.org/article/S0196-6553(16)30223-1/abstract.

32. Appenheimer AB, Diekema DJ, Berriel-Cass D, Crook T, Daley CL, Dobbie D, Edmond M, Hellinger W, Ince D, Julian KG, Lampen R, Arbulu R, Cooper E, Curley E III, De Sanctis J, Freer C, Strong M, Gajurel K, Hasan N, Walker S, Whitener C. Mycobacterium chimaera outbreak response: experience from four United States healthcare systems [Abstract] [Internet]. Open Forum Infect Dis; 2016 [consulté le 16 mars 2017];3(Supp 1);S2392. Disponible à I'adresse : https:// academic.oup.com/ofid/article/2637746/Mycobacteriumchimaera-Outbreak-Response.

33. Tan N, Sampath R, Abu Saleh OM, Tweek MS, Jevremovic D, Alniemi S, Wengenack NL, Sampathkumar P, Badley AD. Disseminated Mycobacterium chimaera infection after cardiothoracic surgery. Open Forum Infect Dis. 2016;3(3):ofw131. Disponible à l'adresse : http://ofid. oxfordjournals.org/content/3/3/ofw131.full.

34. L'ICM avise ses patients opérés à coeur ouvert depuis 2012 d'un risque d'infection [Internet]. Montréal (QC): Institut de Cardiologie de Montréal; 2016 Oct. 24 [consulté le 2017 Mar 16]. Disponible à l'adresse : http://www.icm-mhi.org/fr/ salle-presse/nouvelles/licm-avise-ses-patients-operes-coeurouvert-depuis-2012-dun-risque-dinfection.

35. Garvey MI, Ashford R, Bradley CW, Bradley CR, Martin TA, Walker J, Jumaa P. Decontamination of heatercooler units associated with contamination by atypical mycobacteria. J Hosp Infect. 2016;93(3):229-34. Disponible à I'adresse : http://www.sciencedirect.com/science/article/pii/ S0195670116001274.

36. Hnatuck $P$, Brumbach A, Stolberg DA, Snyder R, Baer L, Mincemoyer S, Whitener C, Julian KG. A case study: discordant results in mycobacterial culturing of cardiac bypass heater-cooler machines_seek and you may find. Am J Infect Control. 2016;44(6, Suppl 2):S50. Disponible à l'adresse : http://dx.doi.org/10.1016/j.ajic.2016.04.042.

37. Gotting T, Jonas D, Scheibert D, Klassen S, Ebner W. Infection risk in cardiothoracic surgery due to contaminated heater cooler units. From 3rd International Conference on Prevention and Infection Control (ICPIC 2015). BioMed Central [Internet]. 2015 4(Suppl 1):73. Disponible à I'adresse : http://www.aricjournal.com/content/4/S1/P73. 